\title{
Magnetic Separation on a New Level: Characterization and Performance Prediction of a cGMP Compliant "Rotor-Stator" High-Gradient Magnetic Separator
}

\author{
Moritz Ebeler, Florian Pilgram, Kai Wolz, Gunnar Grim, and Matthias Franzreb*
}

The growing market of biopharmaceuticals and the constant developments in upstream fermentation have generated a strong demand for new downstream purification methods. Magnetic separation in combination with functional magnetic particles has been known for many years as a promising candidate for a direct capturing tool in protein purification but the lack of suitable GMP-compliant purification equipment has prevented the launch of this technology in large scale bioprocessing. To tackle this bottle-neck, the principle of a "rotor-stator" high-gradient magnetic separator is fully redesigned to meet the rigorous requirements of modern cGMP biotechnology purification processes. In order to fulfill regulatory requirements, the separation chamber is reengineered to allow effective cleaning and sterilization in place while maintaining excellent separation capacities and efficiencies. Two kinds of commercially available magnetic particles are used to validate key performance data and determine system related parameters in order to calculate process performance figures for process optimization of the new magnetic separation device. With separation capacities of over $400 \mathrm{~g}$ of magnetic particles per liter of separation chamber volume and separation efficiencies as well as recovery rates over $99 \%$, the system is able to process more than $200 \mathrm{l}$ crude feedstock per day and capture more than $1.6 \mathrm{~kg}$ target compounds.

expected. $^{[1,2]}$ Alternatives are direct or integrated capturing methods. High gradi ent magnetic separation (HGMS) is an elegant method combining classical purifi cation process steps of solid liquid separa tion, capturing, and concentration in one unit operation. ${ }^{[3-5]}$ HGMS in combination with target selective magnetic particles (MP) enables protein purification directly from unclarified feedstocks. ${ }^{[6]}$ Using small non porous MP prevents fouling due to pore blocking and provides large surface areas, resulting in high binding capacities and fast kinetics. ${ }^{[3]} \mathrm{MP}$ are easy to handle in a batch stirring tank arrangement for the binding step and fast to separate through magnetic force. ${ }^{[7]}$ Magnetic separation has been known for many years in industries such as mining or water recycling. ${ }^{[8-10]}$ HGMS systems were designed to recover micron sized and weakly MP from dry and liquid feed streams. Fixed ferromagnetic matrices, such as filamentary rods, steel wool, thin wire meshes, plates, or pebble beds offer large surface areas while generating high field gradients when placed in a magnetic background field, making them suitable for the different

\section{Introduction}

The large scale purification of biopharmaceuticals from of crude bioprocess feedstock and natural sources by conventional chromatography is often limited. Competing protein concen trations of sometimes over $50 \mathrm{gl}^{-1}$ in serum or fermentation broth homogenates combined with high concentrations of particulate contaminants in the feedstock require elaborate multistep filtration and purification procedures. As a conse quence, low yield, long processing times, and high costs can be

\footnotetext{
M. Ebeler, F. Pilgram, Prof. M. Franzreb

Institute of Functional Interfaces

Karlsruhe Institute of Technology

Eggenstein Leopoldshafen, Germany

E mail: matthias.franzreb@kit.edu

K. Wolz, G. Grim

ANDRITZ KMPT GmbH

Vierkirchen, Germany
}

separation tasks in the mentioned industries. ${ }^{[3,9,11]}$ However, in the pharmaceutical industry, magnetic separation has not yet been used for purification processes on a commercial, industrial scale. ${ }^{[3,4,12]}$ Magnetic separation of biomolecules is mainly employed on a lab scale for cell sorting, DNA purification and analytical applications. ${ }^{[13-15]}$ Nevertheless, there are several studies dealing with the purification of commercially interesting biomolecules via magnetic separation on various scales. ${ }^{[16-19]}$ For this purpose, custom made prototype magnetic separators have been developed with a wide variety of approaches of shape, sizes, and magnetic background field but still following the classical fixed matrix design. Rolled or staged wire meshes or filamentary rod constructions are favored as a defined matrix structure. ${ }^{[20-22]}$ The use of these matrix designs results in good separation performances with over $90 \%$ of MP separation from the feed streams, but the resuspension efficiency needed in order to wash and recover the MP from the system for further use has been mostly ignored. However, the complete recovery of the MP from the separator is essential for economic reasons due 
to the high MP costs and also to avoid batch to batch contaminations. ${ }^{[3,4,15]}$ Only a few approaches for enhanced MP recovery have been described, ranging from high flow rates by circulation of the process fluids over mechanical shaking of the system up to special coatings of the matrix in combination with ultra sonic systems. ${ }^{[23-25]}$ These approaches have been used in $\mathrm{ml}$ scale chamber volumes and face major difficulties in scale up. To overcome disadvantages of the filamentary matrix structures such as irregular shapes and junction points that offer MP and impurities areas of low flow speeds, the matrix design itself has to be optimized. This was done with the development of the "rotor stator" matrix design. ${ }^{[26]}$ The concept of the "rotor stator" magnetic separator is now known in various setups and scales and has proven its broad range of application possibilities in numerous purification processes in different groups. ${ }^{[6,17,19,27]}$ However, these processes have still not been developed past laboratory scale and are still suffering from the lack of suitable large scale GMP conforming magnetic separation equipment. In this work, we present the first GMP compliant high gradient magnetic separa tor suitable for industrial use. The separator follows the concept of the established "rotor stator" high gradient magnetic separator developed in our group previously. ${ }^{[2]}$ Furthermore, we prove the applicability with performance data on separation efficiency as well as separation capacity for two commercially available MP. Additionally, we give an introduction to the process economics of the magnetic separator on the basis of dimensionless key figures describing the purification process in order to show the great potential of the new magnetic separator in a production environment.

\section{Experimental Section}

\section{1. "Rotor-Stator" High-Gradient Magnetic Separator}

Previous HGMS systems typically used filamentary wire meshes as a separation matrix in order to strengthen and concentrate the magnetic field as well as enlarge the separation area for MP. The drawback of this kind of matrix is the lack of cleanability as well as the problems associated with recovery of MP from the system. To overcome this difficulties the "rotor stator" matrix design was developed. The matrix consists of a stack of metal discs (Figure 1C). The discs are densely perforated with holes in order to pump the process solutions through. The metal ligaments between the holes serve as the separation matrix. These areas get highly magnetized to strongly attract MP in the magnetic field of the external electro magnet. The alternating connection of discs to a central rotatable shaft and to the housing of the separation chamber allows a fast rotation of every second disc, while the opposing discs form a stator which stands still (Figure 1B). This arrangement means that high shear forces for particle detachment, resuspension and mixing can be generated in the gap between the rotor and stator discs when required during washing and elution steps. However, existing models of "rotor stator" HGMS systems are not designed to meet GMP guidelines. An open matrix arrangement without a sealing concept to the inner shaft nor the surrounding chamber as well as the $\mathrm{O}$ ring sealing at all chamber openings and metal metal contacts create dead volumes and therefore pose high risk areas for the cleaning or sterilization of the device. Furthermore, the separation chamber, hose connections and valves are not designed to be self draining. This effect is additionally reinforced by the surface and weld quality of the matrix and separation chamber. Finally, the housing of the separation device is not meant to be cleaned. In order to meet demanding requirements of industrial GMP complaint equipment and develop a device which is cleanable (CIP) and sterilizable (SIP) in place, the design of the existing system was fully reengineered in a close cooperation between the company Andritz KMPT GmbH and the Karlsruhe Institute of Technology.

This newly developed fully capsulated version of a "rotor stator" magnetic separator (Figure 1A) meets common hygienic design standards such as 3 A Sanitary Standards or EHEDG and is suitable for applications in clean rooms up to class 7. All surfaces are cleanable and there is no hazard associated with leaching of components into the products. Surface finishes of at least $0.8 \mu \mathrm{m}$ Ra with minimum radii of $3 \mathrm{~mm}$ and electro polished matrix elements are additionally realized. ${ }^{[28]}$ Metal metal contacts are avoided and the system is designed to be fully self draining as well as CIP and SIP compatible. The separator includes of a two way hose pump with a maximum capacity of $160 \mathrm{dm}^{3} \mathrm{~h}^{-1}$. The fluid streams are controlled by two CIP and SIP enabled multi port diaphragm valve blocks (GEMÜ, Ingelfingen Criesbach, Germany) with six connections on the top and bottom of the system which are EHEDG certified. In this system, a switchable electromagnet generates the magnetic field for the separation of MP. The magnet consists of an aluminium winding coil generating a field strength of at least 0.25 Tesla at a power input of $3.6 \mathrm{~kW}$. The impact of the matrix on the magnetic field inside the chamber was simulated by Multiphysics Modeling Software (COMSOL, Goettingen, Germany). The simulation of the field strength shows a magnetic induction up to 0.6 Tesla with the separation matrix installed and a macroscopically homogenous magnetic field over the whole length of the separation chamber. A water cooling jacket comprising the top and bottom lid as well as the inner bore of the magnet controls the temperature which is monitored by a Pt 100 element at the outer winding. The separation chamber has an inner volume of approximately one liter and contains the alternating stack magnetizable filter discs illustrated in Figure 1B. The discs are made from magnetizable stainless steel with an electro polished surface finish. The rotatable discs can be moved at 1,500 rpm. Discs are sealed to each other in order to provide a defined and closed process chamber. For this purpose, custom made PEEK elements were developed. The sealing is designed to avoid any dead spaces and reduce bypass flow around the discs. The inlet and outlet of the chamber allow a plug flow geometry as well as an optimal emptying of the chamber. All parts of the separator which are in contact with product are constructed to be sterilizable with superheated steam. To compensate for the extension of the disc stack and PEEK sealings due to temperatures of up to $125^{\circ} \mathrm{C}$ during the sterilization the process chamber is equipped with pre loaded springs at the top and bottom. In order to avoid O ring sealing and the associated cleaning problems, a double acting mechanical seal is used as shaft sealing. The mechanical seal consists of non magnetizable stainless steel to avoid any collection of MP; furthermore the seal is sterilizable from both 


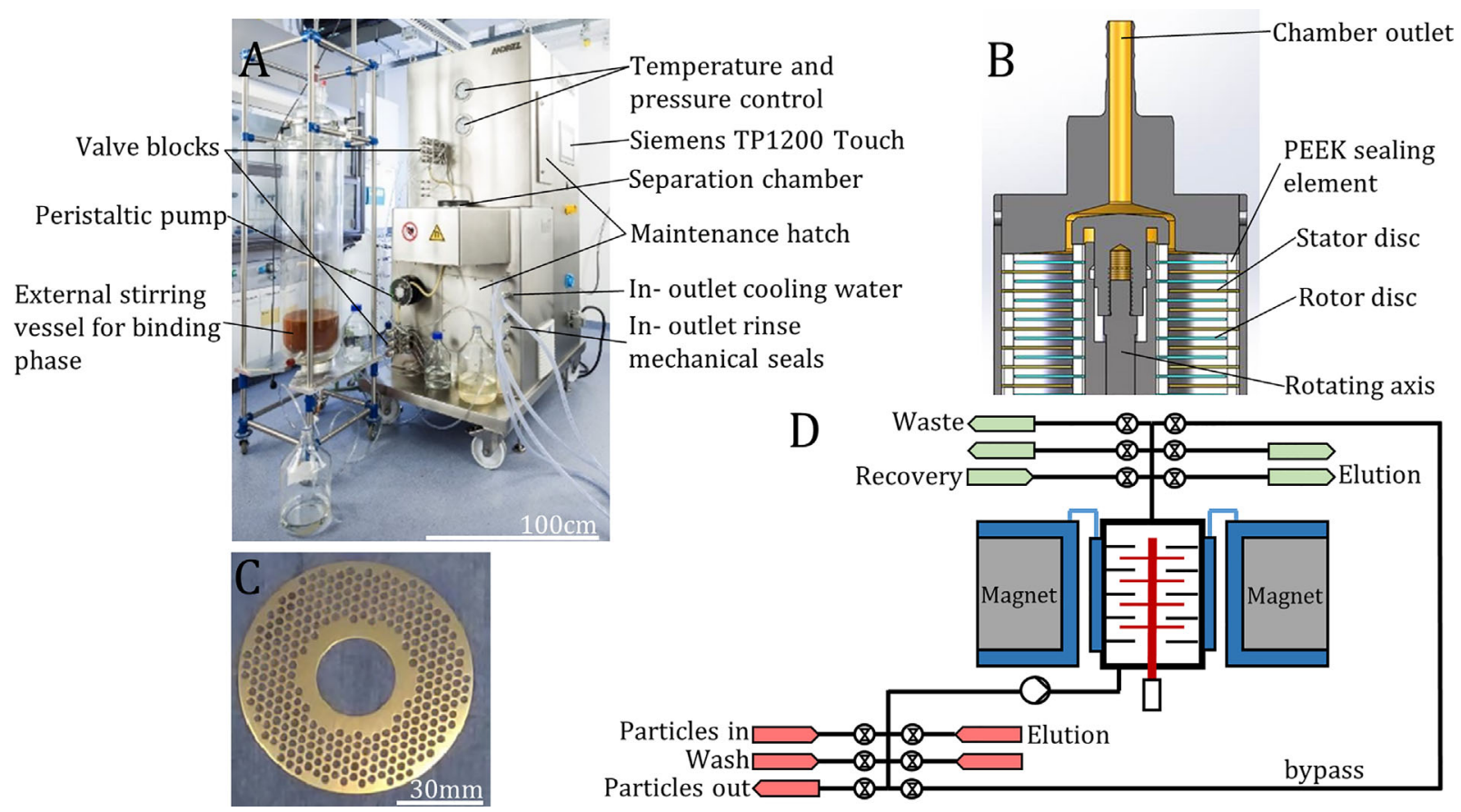

Figure 1. A) Annotated photograph of the "rotor stator" high gradient magnetic separator MES 100 RS, including external stirring vessel for batch adsorption; (B) CAD drawing of the upper part of the separation chamber; (C) perforated separation matrix rotor disc; (D) schematic drawing of the separator including top and bottom valve block with indications of connected feed streams and flow path.

sides. Important system parameters such as temperature and pressure of the sealing liquid as well as core temperature of the magnet are monitored and displayed during the entire separation process. The maintenance requirements of the device are minimized by its design. Tubing can be easily connected via hose nozzles and due to the avoidance of grinding by using mechanical seals the system does not have to be disassembled frequently in order to replace wearing parts. To access the separation matrix, the separation chamber consisting of matrix discs, PEEK sealing, spring packs and a housing can be easily removed from the bore of the magnet in one piece.

The system can be fully controlled and programmed from a human interface type Siemens TP1200 Touch integrated into the housing of the separator. The software is based on a Simatic S7 PLC interface programmed to be highly modular. The operator is flexible in the process design and execution. It is possible to choose from different operating modes such as a manual mode where the operator controls all functions in real time or a fully automated mode where the system runs purification protocols independently.

\subsection{Magnetic Particles}

Two commercially available MP were used to test the performance of the developed HGMS system:Chemagen $M$ PVA MP from PerkinElmer (Waltham, Massachusetts, United States) and MagPrep Silica MP from Merck Millipore (Darm stadt, Germany). The M PVA particles consist of nano sized magnetite particles surrounded by a matrix of cross linked polyvinyl alcohol. According to the manufacturer, the mean diameter ranges from 1 to $3 \mu \mathrm{m}$. The saturation magnetization, determined with an alternating gradient magnetometer (Micro mag 2900 Princeton Measurements) amounts to $29.6 \pm 0.4 \mathrm{Am}^{2}$ $\mathrm{kg}^{-1}$ with a remanence of $14.8 \mathrm{~mA}^{2} \mathrm{~kg}^{-1}$. MagPrep particles consist of monocrystalline magnetite with a thin silica coating. The mean diameter specified by the manufacturer is 100 $200 \mathrm{~nm}$. These particles have a saturation magnetization of $77.6 \pm 3 \mathrm{Am}^{2} \mathrm{~kg}^{-1}$ and a remanence of $25.6 \mathrm{Am}^{2} \mathrm{~kg}^{-1}$.

The particle concentration of collected particle suspensions was determined gravimetrically by dry mass. ${ }^{[19]}$ For concentrations lower than $0.2 \mathrm{gl}^{-1}$ the concentration was determined by absorbance which is linearly dependent on concentration at a wavelength of $860 \mathrm{~nm}$. In this case the samples were transferred to a microtiter plate and measured with a grid of 21 measuring points in triplicates using a plate reader (EnSpire Multimode, PerkinElmer).

\subsection{Operating Procedure}

All operation protocols were programmed and executed via the integrated control panel. Feedstocks containing MP were stirred constantly to ensure a homogenous particle distribution. In order to test the maximum filter capacity of the magnetic separator, MP suspensions with a concentration of $22 \mathrm{gl}^{-1}$ for M PVA MP and $20 \mathrm{gl}^{-1}$ for MagPrep MP were prepared with PBS (137 mM NaCl, $2.7 \mathrm{mM} \mathrm{KCl}$, and $12 \mathrm{mM}$ Phosphate, $\mathrm{pH} 7.4$ ) as liquid phase. A $35 \%(\mathrm{w} / \mathrm{w})$ sucrose solution was used to increase the viscosity of the feed solution.

For separation capacity tests, an MP suspension was pumped at $2.2 \mathrm{dm}^{3} \mathrm{~min}^{-1}$ through the separator from the bottom valve block to the top (Figure 1D) while the electro magnet was 
switched on. Samples were drawn every $15 \mathrm{~s}$ and the particle concentration was determined.

In order to test MP loss after resuspending and recapturing of MP, $90 \mathrm{~g}$ of M PVA MP were loaded into the system. To perform a recirculation of particles as it occurs during buffer change or washing of the MP the system was flushed with two liters of the new buffer system. In the next step, the MP were resuspended by switching off the electro magnet and rotating of the central shaft with the connected discs at $1500 \mathrm{rpm}$ for $30 \mathrm{~s}$. For recapturing MP after suspension, the magnet was switched on again and MP were pumped through the system in a loop from the top to bottom valve block at $1.4 \mathrm{dm}^{3} \mathrm{~min}^{-1}$ for $30 \mathrm{~s}$ in order to collect all MP at the separation matrix. Samples were drawn from the effluent of every buffer change of ten consecutive process cycles.

To recover MP from the chamber, the electromagnet was switched off and the particles were suspended by rotating the central shaft. Different recovery protocols were tested (data not shown). The lowest recovery volume, which represents the ideal case, was achieved by flushing the system from top to bottom with recovery buffer at $100 \%$ pump speed and a rotator speed of $150 \mathrm{rpm}$. The first two fractions contained two liters; for all following fractions the volume was reduced to one liter of buffer.

\section{Results}

\subsection{Separation Performance}

In this study, the performance of the new HGMS device was tested in regards to separation performance, recyclability and recovery of MP. For successful process development, crucial particle dependent parameters were investigated. Separation capacity of the filter matrix was determined by breakthrough experiments at a constant flow rate of $90 \mathrm{dm}^{3} \mathrm{~h}^{-1}$. For M PVA MP, the average filter capacity until a $1 \%$ breakthrough of the feed concentration was $430 \mathrm{~g}$ of MP. Running the system at $90 \mathrm{dm}^{3} \mathrm{~h}^{-1}$ and $22 \mathrm{gl}^{-1}$ feed particle concentration led to a separation duration of $8 \mathrm{~min}$ and $54 \mathrm{~s}$. During this time, an average separation efficiency of $99.91 \%$ was achieved. Separation of M PVA MP from a feed solution with higher viscosity led to the slightly lower separation capacity of $395 \mathrm{~g}$ and less sharp breakthrough behavior, resultiong in a lower separation efficiency of $99.82 \%$. For the smaller MagPrep MP breakthrough was observed after separation of $270 \mathrm{~g} \mathrm{MP}$, within $6 \mathrm{~min}$ and $8 \mathrm{~s}$. The average separation efficiency for this beads was $99.98 \%$, indicating a very sharp breakthrough behavior (Figure 2A).

The combined particle loss of the system during ten suspension and recapturing steps, representing a complete protein purification operation including all washing, elution and regeneration steps, accounted for $0.3 \%$ of the total loaded particle mass for M PVA and $0.2 \%$ for MagPrep MP.

Finally, results from particle recovery experiments for the two kinds of MP are displayed in Figure 2B. Three recovery steps were needed to recover $99.6 \%$ of the loaded M PVA MP. Nearly all particles were found in the first two liters of the first step. No MP were detected in the drain of the fifth and subsequent recovery steps or in the separation chamber after dissembling. However, six recovery steps were required to recover $99.8 \%$ of the MagPrep MP.

\subsection{Process Performance}

As a model system and in order to predict the performance of the new magnetic separation device data obtained by the use of commercial M PVA MP (\#IDA2_0118071) with covalently bound iminodiacetic acid groups charged with $\mathrm{Cu}^{2+}$ ions were used to simulate the large scale purification of his tagged green fluores cent protein (his GFP). In lab scale experiments, the particles showed a maximum binding for his GFP directly from the unclarified E.coli cell lysate of $Q_{\text {max }}: 0.168 \mathrm{gg}^{-1}$ with a $K_{\mathrm{d}}$ of $0.063 \mathrm{gl}^{-1}$. For the following calculations the particle mass $\mathrm{m}_{\mathrm{p}}$ was set to $316 \mathrm{~g}$, accounting for $80 \%$ of the $1 \%$ breakthrough for high viscous feedstocks. The separator volume $V_{\text {sep }}$ is fixed at 0.981 and the initial his GFP concentration is set to $8 \mathrm{gl}^{-1}$. With a variation of the capacity ratio CR which involves a variation of $V_{\text {batch }}$ the equilibrium concentration $c^{*}$ can be calculated. As described, protein purification by MP in combination with a magnetic separation device uses a batch binding step in a stirred external tank. Consequently, the achievable protein loading of the MP depends on the remaining protein concentration in the superna tant after equilibrium is reached. This results in the known interrelation that high binding yields (low equilibrium concen trations left in solution) correspond to low protein loadings of the MP and vice versa. The equilibrium conditions are influenced by the ratio of the protein amount that can be bound by the mass of MP used and the protein amount offered in the feed volume of the batch. In previous publications we showed how this CR and the isotherm parameters of the considered purification task influence the predicted yield, purity and productivity of protein purification using magnetic separation ${ }^{[7]}$ (see supporting information). Productivity and resulting yield of the described model process are illustrated in dependence of the CR in Figure 3. The yield increases up to $95 \%$ in a linear manner up to a CR of approx. 1, after which the further yield increase is quite small with increasing CR. In the case of MP with high target affinity, the productivity also reaches its maximum close to this point, in our case at a CR of 1.1, corresponding to a batch volume of approx. $5.5 \mathrm{dm}^{3}$. To process the batch volume, a cycle time of $37 \mathrm{~min}$ has been experimentally determined. The cycle time is mainly influenced by the pump speed and includes the time for all process steps, such as loading, washing, elution, particle cleaning, equilibration and recovery. In summary, in the case of the described model system, a yield of $96.7 \%$ is predicted with a productivity of $1.17 \mathrm{~g} \mathrm{~min}^{-1 *} \mathrm{~L}$. In $24 \mathrm{~h}$ the simulated process is able to treat 2101 of fermentation broth while capturing and purifying more than $1.6 \mathrm{~kg}$ of his GFP.

\section{Discussion}

The separation performance test with the novel GMP complaint "rotor stator" magnetic separator revealed that separation capacity as well as breakthrough behavior strongly depends on the properties of the MP. Decisive in this regard are the significant differences in size, magnetization, and agglomera tion properties. Despite the high magnetization, the size and weight of the MagPrep MP is crucial for the separation step. For protein purification processes, the particle amount loaded to the separator should not exceed $80 \%$ of the maximum loading to ensure an optimal washing of the MP in the chamber. The 

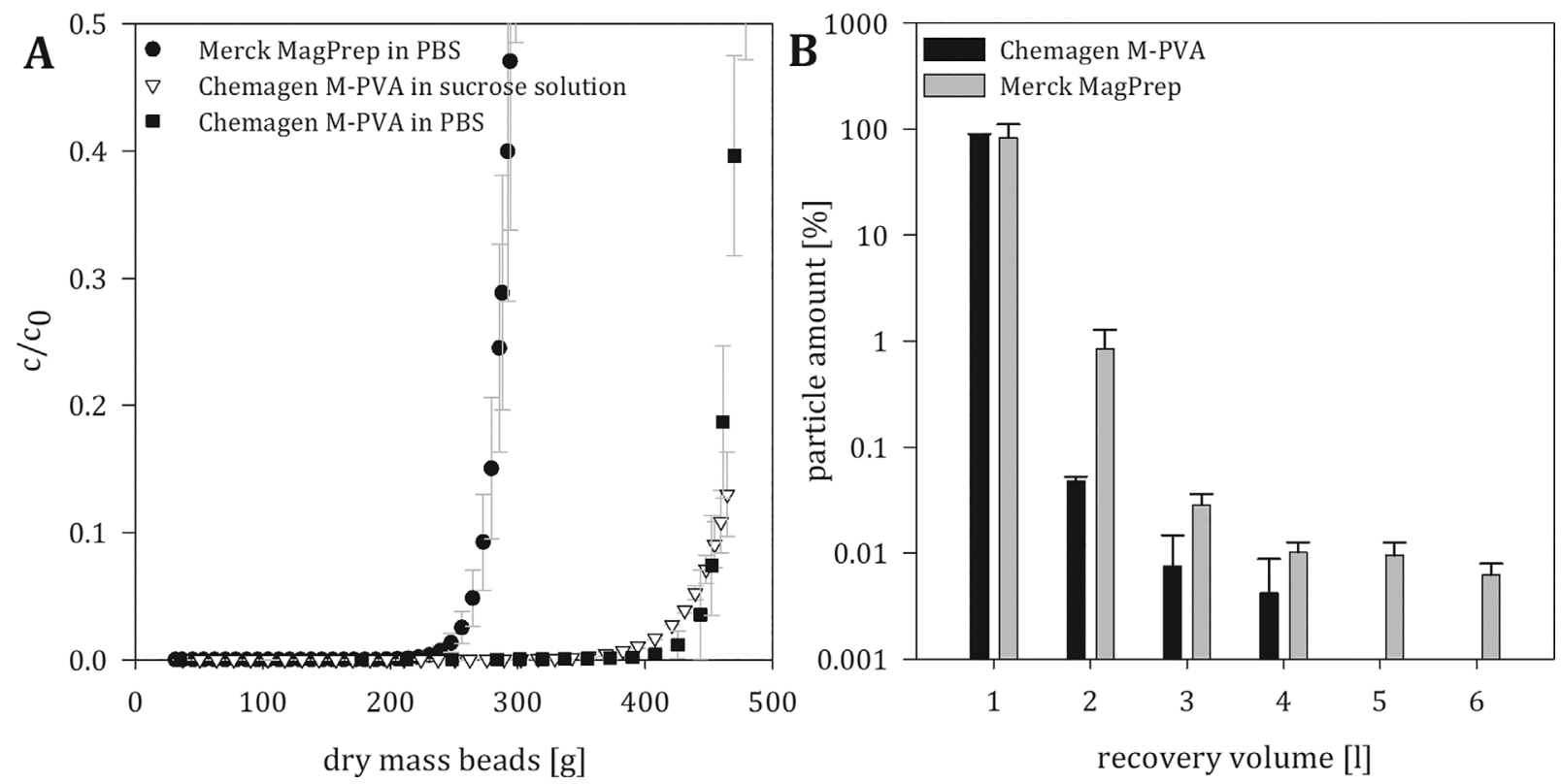

Figure 2. A) Breakthrough curves for two kinds of commercial MP. Dots: Merck MagPrep MP; Inverse triangle: Chemagen M PVA MP in 37\% (w/w) sucrose solution; Squares: Chemagen M PVA MP. B) Recovery volumes for two kinds of commercial MP. Y axis: Fraction of MP in per cent of the total loaded amount. X axis: Number of applied 1 I batches of recovery solution. In black bars, Chemagen M PVA MP and in grey Merck MagPrep MP.

viscous feed solution was chosen to simulate natural and sticky feedstocks such as blood serum. The shearing effect of the viscous feed solution resulted in a lower separation capacity as well as efficiency. Lower pump speeds should allow the particles to settle fast enough on the separation matrix and achieve separation results comparable to the ones with aqueous feed solutions. The separation device presented here meets or even exceeds ideal specifications for a magnetic separation device compiled by Franzreb et al. ${ }^{[3]}$ At the moment there is no comparable system on this scale on the market. Separation efficiencies are comparable to smaller systems optimized for

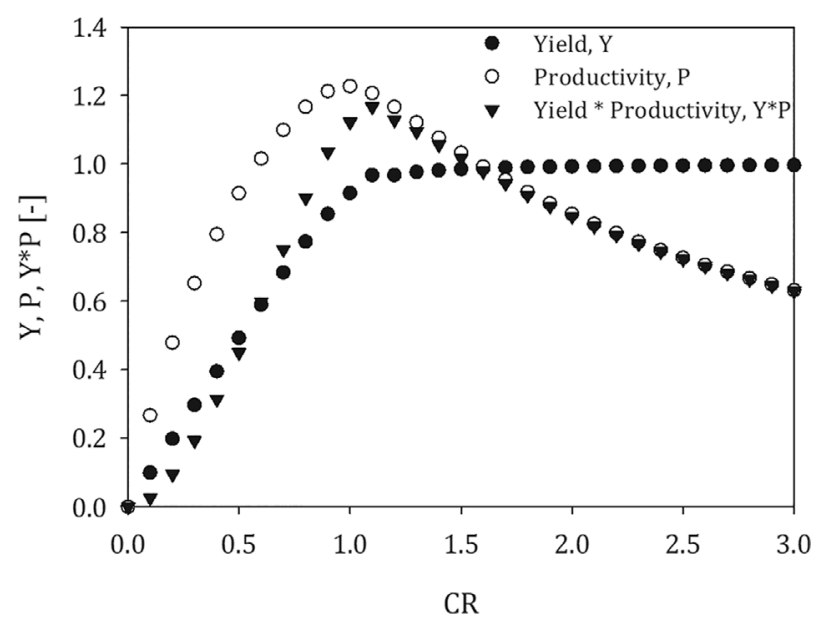

Figure 3. Dots: Yield; inverse triangle: Productivity; squares: Yield*Pro ductivity as function of the capacity ratio CR for the target protein his GFP. Yield*Productivity depending on CR indicates an optimal process point at CR 1.2. particle separation without taking GMP guidelines and cleanability into account. ${ }^{[19,23]}$ The outstanding MP recovery performance due to the matrix design allows an economic process design with optimal MP washing results during the process. Furthermore, theoretical results of the process simula tion are in good correlation with process results for a his GFP purification using a previous version of a "rotor stator" magnetic separator from Gracìa et al. ${ }^{[19]}$ The simulation in combination with the results for the two strongly differing types of MP show the high efficiency and flexibility of the developed magnetic separator. Nevertheless, suitable MP have to be chosen carefully for every process. Besides the affinity and selectivity for the target molecule, the magnetization, size as well as size distribution, structure and availability are crucial factors.

\section{Conclusions}

In this work, a GMP compliant "rotor stator" high gradient magnetic separator ready for the use in biopharmaceutical purification processes was introduced. The device, whose commercialization was launched at the beginning of the year 2017, allows the expansion of applications for magnetic separation from analytic and small laboratory scale to the integration of this elegant direct capturing tool to biopharma ceutical production processes. An approved matrix design combined with a reengineered separation chamber and an advanced sealing concept showed excellent separation capacities while overcoming difficulties in MP recovery and cleanability of previous designs of magnetic separators. It was shown that the breakthrough behavior of MP is widely independent of the solution but strongly depends on the type of MP. Simulated operation data based on small scale experimental results showed 
promising process performance, capable of treating more than 2001 of crude fermentation broth a day with the direct capturing unit operation presented here.

\section{Abbreviations}

GMP, good manufacturing practice; HGMS, high gradient magnetic separation; MP, magnetic particles; CIP, cleanable in place; SIP, sterilizable in place; PEEK, polyether ether ketone; EHEDG, European Hygienic Engineering and Design Group.

\section{Supporting Information}

Supporting information is available from the Wiley Online Library or from the author.

\section{Acknowledgements}

The authors sincerely acknowledge Kathryn Melzak and Lisa Rödling for proofreading this manuscript. This work was carried out within the project ProSeCa and was financed by BMBF (031A340A)

\section{Conflict of Interest}

The authors declare no financial or commercial conflict of interest.

\section{Keywords}

direct capturing, downstream processing, GMP, high gradient magnetic separation, industrial biotechnology

[1] A. L. Zydney, Biotechnol. Bioeng. 2016, 113, 465.

[2] B. Kelley, Biotechnol. Prog. 2007, 23, 995.
[3] M. Franzreb, M. Siemann Herzberg, T. J. Hobley, O. R. T. Thomas, Appl. Microbiol. Biotechnol. 2006, 70, 505.

[4] L. Borlido, A. M. Azevedo, A. C. A. Roque, M. R. Aires Barros, Biotechnol. Adv. 2013, 31, 1374.

[5] J. Hubbuch, D. B. Matthiesen, T. J. Hobley, O. R. T Thomas, Bioseparation. 2001, 10, 99.

[6] G. N. Brown, C. Müller, E. Theodosiou, M. Franzreb, O. R. T. Thomas, Biotechnol. Bioeng. 2013, 110, 1714.

[7] A. A. Shukla, M. R. Etzel, S. Gadam, Process Scale Bioseparations for the Biopharmaceutical Industry. CRC Press, FL, USA 2006.

[8] M. Franzreb, W. H. Höll, Trans. Appl. Supercond. 2000, 10, 923.

[9] J. Oberteuffer, IEEE Trans. Magn. 1974, 10, 223.

[10] J. H. P. Watson, D. C. Ellwood, IEEE Trans. Magn. 1987, 23, 3751.

[11] J. Oberteuffer, IEEE Trans. Magn. 1973, 3, 167.

[12] W. Li, L. Yang, F. Wang, H. Zhou, H. Xing, X. Li, H. Liu, Ind. Eng. Chem. Res. 2013, 52, 4290.

[13] G. M. Whitesides, R. J. Kazlauskas, Trends Biotechnol. 1983, 1, 144.

[14] B. I. Haukanes, C. Kvam, Nat. Biotechnol. 1993, 11, 60.

[15] I. Safarik, M. Safarikova, Biomagn. Res. Technol. 2004, 2, 7.

[16] K. Holschuh, A. Schwämmle, J. Magn. Magn. Mater. 2005, 293, 345.

[17] J. Hubbuch, O. R. T. Thomas, Biotechnol. Bioeng. 2002, 79, 301.

[18] C. Müller, E. Heidenreich, M. Franzreb, K. Frankenfeld, Biotechnol. Prog. 2014, 31, 78.

[19] P. F. García, M. Brammen, M. Wolf, S. Reinlein, M. Freiherr von Roman, S. Berensmeier, Sep. Purif. Technol. 2015, 150, 29.

[20] A. Pasteur, N. Tippkötter, P. Kampeis, R. Ulber, IEEE Trans. Magn. 2014, 50, 1.

[21] N. A. Ebner, T. J. Hobley, O. R. T. Thomas, M. Franzreb, IEEE Trans. Magn. 2007, 43, 1941.

[22] A. Heebøll Nielsen, W. S. Choe, A. P. J. Middelberg, O. R. T. Thomas, Biotechnol. Prog. 2003, 19, 887.

[23] H. C. Roth, A. Prams, M. Lutz, J. Ritscher, M. Raab, S. Berensmeier, Chem. Eng. Technol. 2016, 39, 469.

[24] A. Heebøll Nielsen, S. F. L. Justesen, T. J. Hobley, O. R. T. Thomas, Sep. Sci. Technol. 2004, 39, 2891.

[25] A. Heebøll Nielsen, M. Dalkiær, J. Hubbuch, O. R. T. Thomas, Biotechnol. Bioeng. 2004, 87, 311.

[26] M. Franzreb, C. Reichert, Hochgradienten Magnetabscheider, DE Patent 102004034541, 2006.

[27] C. Müller, K. Wagner, K. Frankenfeld, M. Franzreb, Biotechnol. Lett. 2011, 33, 929.

[28] G. Hauser, G. J. Curiel, H. W. Bellin, H. J. Cnossen, J. Hofmann, EHEDG Guidelines 2004, 2, 16. 
Karlsruher Institut für Technologie

\section{Repository KITopen}

Dies ist ein Postprint/begutachtetes Manuskript.

Empfohlene Zitierung:

Ebeler, M.; Pilgram, F.; Wolz, K.; Grim, G.; Franzreb, M.

Magnetic Separation on a New Level : Characterization and Performance Prediction of a cGMP Compliant 'Rotor-Stator' High-Gradient Magnetic Separator.

2018. Biotechnology journal, 13

doi:10.5445/IR/1000076326

Zitierung der Originalveröffentlichung:

Ebeler, M.; Pilgram, F.; Wolz, K.; Grim, G.; Franzreb, M.

Magnetic Separation on a New Level : Characterization and Performance Prediction of a cGMP Compliant 'Rotor-Stator' High-Gradient Magnetic Separator.

2018. Biotechnology journal, 13 (2), Article No.1700448.

doi:10.1002/biot.201700448 